\title{
A list of new nearby dwarf galaxy candidates ${ }^{\star}$
}

\author{
V.E. Karachentseva ${ }^{1,2}$ and I.D. Karachentsev ${ }^{1,3}$ \\ 1 Visiting astronomer, Astrophysikalisches Institut Potsdam, an der Sternwarte, 16 D-14482, Potsdam, Germany \\ 2 Astronomical Observatory of Kiev University, Observatorna, 3, 254053, Kiev, Ukraine \\ ${ }^{3}$ Special Astrophysical Observatory, Russian Academy of Sciences, N.Arkhyz, Stavropolsky Kraj, 357147, Russia
}

Received February 6; accepted May 29, 1997

\begin{abstract}
To increase completeness of the distance limited sample of nearby galaxies from the Kraan-Korteweg \& Tammann (1979) catalogue we undertook a search for small companions of larger known galaxies which have corrected radial velocities within $500 \mathrm{~km} / \mathrm{s}$. Based primarely on the POSS-II and ESO/SERC films we found 260 nearby dwarf galaxy candidates with angular diameters $a \gtrsim 0.5$ arcmin. More than $50 \%$ of the objects were revealed for the first time. As we suppose, a significant part of them (about 30\%) may really belong to the Local Volume sample.
\end{abstract}

Key words: galaxies: distances and redshifts — galaxies: irregular

\section{Introduction}

In a study of the general properties and evolution of galaxies it is necessary to have quite a complete and representative sample biased minimally by observational selection. A catalogue of all nearby galaxies with distances $D<D_{\max }$ would be an ideal realization of such "fair" sample. To be representative such a sample must contain at least $(100-300)$ objects, which can be achieved at $D_{\max }=(5-10) \mathrm{Mpc}$. The knowledge of distances for all nearby galaxies permits both local dynamics and the shape of the gravitational potential field to be evaluated.

Unfortunately, the distances remain one of the most deficient observing parameters for the galaxies even in the vicinity of the Local Group. Being short of distance data, Kraan-Korteweg \& Tammann (1979) compiled a catalogue of nearby galaxies with radial velocities $V_{0}$ (corrected for solar motion) not exceeding $V_{\max }=500 \mathrm{~km} / \mathrm{s}$. Their sample $(=\mathrm{KKT})$ contains 179 galaxies (after excluding probable Virgo cluster members which satisfy the condition

\footnotetext{
* Tables 1 and 2 also available in electronic form at CDS via anonymous ftp to cdsarc.u-strasbg.fr (130.79.128.5) or via ftp://cdsweb.u-strasbg.fr/Abstract.html
}

$V_{0}<500 \mathrm{~km} / \mathrm{s}$ due to large peculiar velocities). Then Huchtmeier \& Richter (1988, 1989a) and Karachentsev (1994) increased the sample in the number to $N=215$ by including new objects detected in the HI $21 \mathrm{~cm}$ line by different authors. Independent efforts to extend the KKTsample were undertaken by Schmidt \& Boller (1992). However, their catalogue of 289 nearby galaxies contains many questionable cases when the radial velocity refers to a globular cluster or a star projected on a distant galaxy. Moreover, the catalogue of Schmidt \& Boller (1992) includes about 40 galaxies without radial velocity, assuming their membership in the nearby groups, but later HI observations did not confirm it.

The galaxy number distribution of the KKT- sample with radial velocities as well as angular diameters show that its expected completeness is $(40-60) \%$ only. For instance, a median value of the linear diameter of the Local Group galaxies equals $0.9 \mathrm{kpc}$ (Karachentsev 1996). Being at $D=3.5 \mathrm{Mpc}$ (the distance of the nearest groups M 81 and Cent.A), a "median" dwarf system has an angular diameter $a=0.9$ arcmin. In fact a lot of the faintest objects in the KKT- sample are represented by the galaxies from the catalogues: DDO (van den Bergh 1966), UGC (Nilson 1973), and ESO/Uppsala (Lauberts 1982) with a limiting angular diameter $a=1$ arcmin. So, we expect that about $(100-250)$ smaller galaxies really situated at $D=4-6 \mathrm{Mpc}$ have not yet been covered by radial velocity surveys (and even have not yet been discovered). The situation is complicated by the presence of the "Zone of avoidance" because of the light extinction in the Milky Way plane.

As experience of the last two decades have shown, the increase in the number of the known galaxies with $V_{0}<500 \mathrm{~km} / \mathrm{s}$ grows slowly (with a rate of $2-3$ galaxies per year) and a discovery of very nearby galaxies happens in fact by chance. That is a reason for us to make special searches for the nearby dwarf galaxy candidates. We consider this work a part of a more extended program of studying the Local Volume galaxies (Karachentsev 1994). 

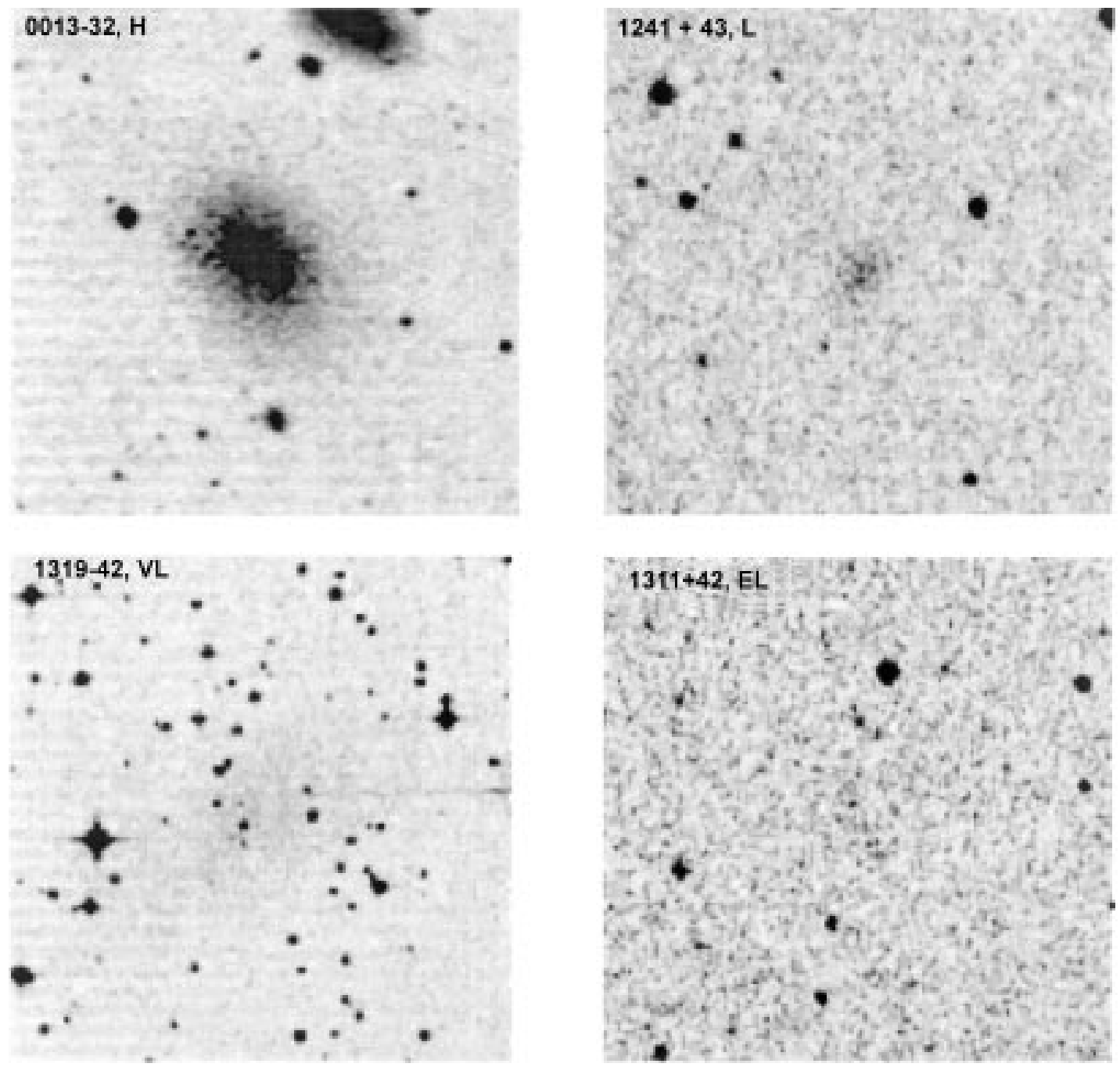

Fig. 1. Reproductions from the digital POSS-I of the objects: $0013-32(\mathrm{SB}=\mathrm{H}), 1241+43(\mathrm{SB}=\mathrm{L}), 1319-42(\mathrm{SB}=\mathrm{VL})$, and $1311+42(\mathrm{SB}=\mathrm{EL})$. Each chart is $4 \times 4$ arcmin, North at top, East at left

\section{Approach to the search}

The availability of high-quality Sky Surveys, POSS-II and ESO/SERC, made on new photographic emulsions stimulated strongly the present search. The high angular resolution and the sensitivity to low contrast details permit the detection and classification with confidence of galaxies of small angular dimensions, especially low surface brightness objects. Using our previous experience of searching for low surface brightness objects on the POSS-I prints (Karachentseva 1968, 1972, 1973), we undertook analogous searches based on new observational material. Due to the different morphology of dwarf galaxies there are no distinct criteria to distinguish them from a normal distant galaxy of the same angular diameter (or from a part of a reflecting nebula). Typical cases of such visual misclassification were described by Karachentseva et al. (1985). Indeed, a search for the nearby dwarf galaxy candidates seems to be a rather delicate subject. To complete the
KKT- sample with new very nearby galaxies we used the following strategy:

1. Dwarf companions of the known nearby galaxies were assumed to be the main supplier of new objects for the Local Volume $(=\mathrm{LV})$. Following this idea we searched on the POSS-II or ESO/SERC two-colour films for dwarf galaxies down to the limiting angular diameter 0.5 arcmin around each of $215 \mathrm{LV}$ galaxies with $V_{0}<500 \mathrm{~km} / \mathrm{s}$ from the list by Karachentsev (1994), which is an up-dated version of the KKT-sample. The diameter of the inspected area around each of the LV galaxies was chosen equal to 50 times its diameter. So, for a normal galaxy $20 \mathrm{kpc}$ in diameter the survey area corresponds to a $1 \mathrm{Mpc}$ diameter circle and exceeds the crossing length for the satellite during a cosmological time of $1 / \mathrm{H}$. Because of the present incompleteness of the POSS-II survey we used in $\sim 15 \%$ of the cases the POSS-I prints too. 
2 . In addition to the inspected neighbourhood of individual galaxies we carried out a total visual examination of wide regions of the well-known nearby galaxy groups: the M 31, M 81, M 101, IC 342/Maffei, Sculptor, Centaurus and the Canes Venatici cloud, indicated in the Nearby Galaxy Catalog of Tully (1988). In case of CnV the survey area was limited to R.A. $=\left[11.5^{\mathrm{h}}, 14.5^{\mathrm{h}}\right]$, D. $=\left[+20^{\circ}\right.$, $+60^{\circ}$.

3. Using the POSS-II and ESO/SERC films we revised the images of dwarf galaxies from the catalogues of Karachentseva \& Sharina (1988), and Arp \& Madore (1989). The objects with signs of possible resolution into stars have been included, as a rule, in the candidate list.

4. To fill in the empty cone evoked by excluding all galaxies in the Virgo cluster direction, we inspected the galaxy images of the angular diameter $a \gtrsim 0.5$ arcmin within the boundaries R.A. $=\left[11.5^{\mathrm{h}}, 13.5^{\mathrm{h}}\right]$, D. $=\left[0^{\circ}\right.$, $+20^{\circ}$. As an example, we considered the dwarf system GR 8 (Reaves 1983) situated in front of the Virgo cluster.

5. Into our list of the LV candidates there were included also galaxies with recent radial velocity determinations, mainly from the HI surveys (Huchtmeier \& Richter 1989b; Shombert et al. 1992; Kraan-Korteweg et al. 1994; Garcia et al. 1994; Gallagher et al. 1995; Huchtmeier et al. 1995; Mattews et al. 1995; Huchtmeier \& van Driel 1996 Mattews \& Gallagher 1996) when their corrected velocities did not exceed $500 \mathrm{~km} / \mathrm{s}$. Note that due to the influence of Galactic hydrogen, the low signal-to-noise ratio or other reasons the small value of galaxy radial velocity would often seem doubtful. That is why our decision to include a galaxy in the list took into account the visual impression about its structure. We used also the available version of the ZCAT (Huchra 1995) which contains 386 objects with $V_{0}<500 \mathrm{~km} / \mathrm{s}$. Of them 215 are in the Karachentsev (1994) list, and 97 objects are members of the Virgo cluster. The remaining 74 ones formed a set of strange cases. Note some typical examples:

- the radial velocity refers to a bright star projected on a distant galaxy (for example, $0232+3725$ ),

- the object is a globular cluster, not a galaxy (NGC 2420),

- the velocity belongs to a HII region in M33 or another large galaxy $(0130+3041)$,

- the velocity belongs to the Galactic hydrogen in the empty area $(0106+2137)$,

- the object is a planetary nebula (NGC 5844) or a cometary nebula $(2022+3541)$,

- the small value of the galaxy radial velocity is a result of misprint $(2318-4200)$.

\section{The list of candidates}

The results of our search for new dwarf galaxy candidates are presented in Table 1 where Cols. 1, 2 are the equatorial coordinates (epoch 1950.0); Cols. 3, 4 are the major and minor angular diameters in arcmin measured on

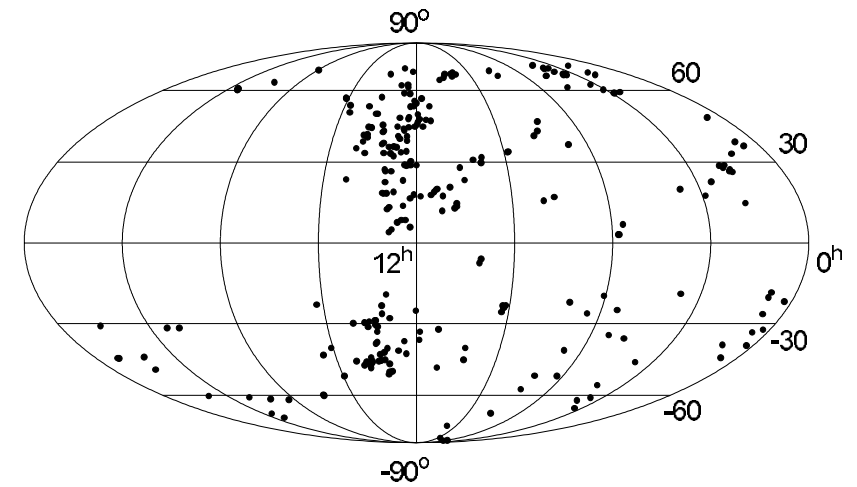

Fig. 2. Distribution of the Local Volume galaxy candidates in the equatorial coordinates

blue films; Col. 5 is the morphological type in usual designations ("d" as a "dwarf" was omitted); Col. 6 is the rough estimate of surface brightness (=SB): H - high (equal or brighter than the SB of a normal spiral galaxy), L - low, VL — very low, EL — extremely low (usually invisible on POSS-I but visible on POSS-II); Fig. 1 illustrates the appearence on the digital POSS-I of four objects with the different SB estimates; Col. 7 is the galaxy name or its number in the catalogues/lists : AM - Arp \& Madore (1987), FG - Feitzinger \& Galinski (1985), K - Karachentseva (1968, 1972, 1973), BK - Börngen \& Karachentseva (1985), DDO — van den Bergh (1966), UGC, UGCA (Nilson 1973, 1974), F — Schombert et al. (1992), VCC - Binggeli et al. (1985), MCG - VorontsovVelyaminov et al. (1962-1968); Col. 8 contains the data on heliocentric radial velocities and galaxy shape and indicates some notes at the end of the Table 1. In preparing this article two HI surveys of nearby galaxies (Huchtmeier et al. 1997; Cote et al. 1997) revealed 15 our objects to have radial velocities greater than $1000 \mathrm{~km} / \mathrm{s}$. A list of them is presented in Table 2 where columns are the same as in Table 1.

The distribution of 260 galaxies from Table 1 is presented in Fig. 2 in equatorial coordinates. Because our survey does not cover the sky continuously, we do not discuss here the details of this distribution. Note only that the presented distribution delineates clearly the concentration toward the Local Supercluster plane and also the wide empty region coinciding with the Local Void (Tully 1988 ) around the direction $\left\{19.0^{\mathrm{h}},+15^{\circ}\right\}$.

\section{Discussion}

As it is seen from Table 1, among 260 galaxy candidates 132 are absent in the previous catalogues and lists. In other words, $51 \%$ of our list objects are new. All but one are visible on two different colour films and we suppose they are real objects. From them 33 galaxies have radial velocity data including 7 recent HI measurements made by Huchtmeier et al. (1997). 
Obviously, there is no direct way to estimate the resulting efficiency of our searches. We did not meet in the literature a similar attempt just concerning the search for very nearby dwarf galaxies. Binggeli et al. (1990) were searching for dwarf galaxies on deep IIIa-J Palomar Schmidt telescope plates in several sky belts in the range of $\mathrm{D}=$ $\left[+28^{\circ},+57^{\circ}\right]$, R.A. $=\left[9^{\mathrm{h}}, 16^{\mathrm{h}}\right]$. Among 179 objects considered by them to be dwarf galaxies there are only 2 objects in common with our list.

Schombert et al. (1992) published a catalogue of LSB galaxies found on the POSS-II survey in the declination zone D. $=\left[0^{\circ},+25^{\circ}\right]$. Their catalogue contains 369 LSB galaxies with angular diameters greater than 0.5 arcmin. Basing on the results of the HI survey of the catalogue galaxies, Schombert et al. (1992) concluded that most of the detected objects are spirals with $V>2000 \mathrm{~km} / \mathrm{s}$. Only 4 dwarf galaxies have $V_{0}<500 \mathrm{~km} / \mathrm{s}$.

Impey et al. (1996) carried out a search for LSB galaxies in the equatorial zone covering an area of 786 sq.degrees. Using a combination of both automated and visual examination of glass copies of the UKST IIIa-J survey plates they detected 516 low surface brightness objects with $a>0.5$ arcmin. For 264 and 160 objects the optical and HI velocities, respectively, have been measured. Our analysis of their catalogue data showed the following:

(a) Only two corrected radial velocities do not exceed the limit of $500 \mathrm{~km} / \mathrm{s}$. But in both cases the measurements refer to stars projected on the galaxies.

(b) There are no common objects in the list of Impey et al. (1996) and our Table 1.

(c) Many Impey et al. (1996) LSB objects of irregular shape on their finding charts, looking like nearby dwarf candidates (for example, 0123-0029, $0129-0024,0217+0031,0224+0238,0224+0233$, $0227+0040$ etc.), are unrecognized absolutely on both (blue and red) films of POSS-II.

In carrying out the search, we found independently all three dwarf spheroidal companions of the M 31: And I, And II, And III which had been discovered by van den Bergh (1972). However, we did not find any new spheroidal nor did we find irregular candidate member of this group. In the zone of strong obscuration in the vicinity of spiral galaxies IC 342 and Maffei 2 we found independently the galaxies Cas 1, Cas 2, MB1 discovered recently by Huchtmeier et al. (1995), Kraan-Korteweg et al. (1994) and McCall \& Buta (1995). Moreover we believe that our list contains probably additional members of this nearby galaxy complex.

It should be emphasized that Table 1 does not contain other nearby galaxies which have been presented in their basic list (Karachentsev 1994). This is the reason why LGS 3, GR 8, K 52, and some other well-known dwarf galaxies do not appear in Table 1.

To clarify the nature of galaxies from our list one needs to measure their radial velocities. The pilot HI survey of the sample of 26 objects from this list made by Huchtmeier et al. (1997) shows that about $70 \%$ of them are detected in the $21 \mathrm{~cm}$ line, and the fraction of very nearby dwarfs with $V_{0}<500 \mathrm{~km} / \mathrm{s}$ consists of $1 / 4$.

\section{Concluding remarks}

Due to the incompletness of the available set of the POSSII films we did not aim at the global task of search for candidates in the sample of galaxies with $D<7 \mathrm{Mpc}$ on the whole sky. The region inspected by us covers about $20 \%$ of the whole sky area. However in our list we expect to see the main part of new members of the Local Volume because our search covers the area occupied by all nearby groups. We suppose that a systematic study of these galaxies in the HI line as well as their multicolour imaging up to a deep limit for photometric distance determinations are tasks of current concern for large radio and optical telescopes.

As regards the theories of galaxy formation and evolution, the search for nearby dwarf galaxies outside galaxy groups is very important too. Especially it is reasonable to reveal dwarf systems in the volume of the Local Void, occupying an area of several steradians on the sky (Tully 1988). A survey of the Local Void region on the POSSII and ESO/SERC is planned as the second part of this project.

Acknowledgements. We are grateful to the director and staff of the Astrophysical Institute Potsdam for hospitality and opportunity to work with the POSS-II Survey. We thank Dr. G.M. Richter for helpful discussion. We are indebted to G.G. Korotkova for the assistance in preparing the manuscript. This project is supported by INTAS-RFBR grant No. 95-INRU-1390.

\section{References}

Arp H.C., Madore B.F., 1987, A Catalogue of southern peculiar galaxies and associations. Cambridge University Press, I, II

Binggeli B., Sandage A., Tammann G.A., 1985, AJ 90, 1681

Binggeli B., Tarengui M., Sandage A., 1990, A\&A 228, 42

Börngen F., Karachentseva V.E., 1985, Astron. Nachr. 306, 301

Cote S., Freeman K.C., Carignan C., Quinn P.J., 1997, AJ (in press)

Feitzinger J.W., Galinski T., 1985, A\&AS 61, 503

Gallagher J.S., Littleton J.E., Matthews L.D., 1995, AJ 109, 2003

Garcia A.M., Bottinelli L., Garnier R., Gouguenheim L., Paturel G., 1994, A\&AS 107, 265

Hopp U., Schulte-Ladbeck, 1991, A\&A 248, 1

Huchra J., 1995, A catalogue of galaxy redshifts

Huchra J.P. Vogeley M.S., Geller M.J, 1997, ApJS (in press)

Huchtmeier W.K., Richter O.-G., 1988, A\&A 203, 237

Huchtmeier W.K., Richter O.-G., 1989a, A\&A 210, 1

Huchtmeier W.K., Richter O.-G., 1989b, A general catalog of HI observations of galaxies. Springer-Verlag, New York, Inc

Huchtmeier W.K., Lercher G., Seeberger R., Sauer W., Weinberger R., 1995, A\&A 293, L33

Huchtmeier W.K., van Driel W., 1996, A\&A 305, L25 
Huchtmeier W.K., Karachentsev I.D., Karachentseva V.E., 1997, A\&A 322, 375

Impey C.D., Sprayberry D., Irwin M.J., Bothun G.D., 1996, ApJS 105, 209

Karachentseva V.E., 1968, Soobsch. Byurakan. Obs. 39, 62

Karachentseva V.E., 1972, Astron.Tzirk. 723, 1

Karachentseva V.E., 1973, Astrofiz. Issled. (Izv. SAO) 5, 10

Karachentseva V.E., Karachentsev I.D., Börngen F., 1985, A\&AS 60, 213

Karachentseva V.E., Sharina M.E., 1988, Catalogue of low surface brightness dwarf galaxies, Comm. Spec. Astrophys. Obs. 57,3

Karachentsev I.D., 1994, A\&Ap Trans. 6, 1

Karachentsev I., 1996, A\&A 305, 33

Kraan-Korteweg R.C., Tammann G.A., 1979, Astron. Nachr. 300, 181

Kraan-Korteweg R.C., Loan A.J., Burton W.B., et al., 1994, Nat 372,77

Lauberts A., 1982, ESO/Uppsala Survey of the ESO(B) Atlas. Garching, European Southern Observatory (ESO)

McCall M.L., Buta R.J., 1995, AJ 109, 2460
Matthews L.D., Gallagher J.S., Littleton J.E., 1995, AJ 110, 581

Mattews L.D., Gallagher J.S., 1996, AJ 111, 1098

Michel A., Huchra J., 1988, PASP 100, 1423

Nilson P., 1973, Uppsala General Catalogue of Galaxies. Uppsala Astron. Obs. Ann. 6 (UGC)

Nilson P., 1974, Catalogue of Selected Non-UGC Galaxies. Uppsala Astron. Obs. Rep. 5 (UGCA)

Reaves G., 1983, ApJS 53, 375

Schmidt K.-H., Boller T., 1992, Astron. Nachr. 313, 189

Schombert J.M., Bothun G.D., Schneider S.E., McGaugh S.S., 1992, AJ 103, 1107

Smoker J.V., Davies R.D., Axon D.J., 1996, MNRAS 281, 393

Tully R.B., 1988, Nearby Galaxy Catalog. Cambridge University Press

van den Bergh S., 1966, AJ 71, 33

van den Berg S., 1972, ApLett. 31, 31

Vorontsov-Velyaminov B.A., Krasnogorskaja A.A., Arkhipova V.P., 1962-1968, The Morphological Catalogue of Galaxies. Moscow State University, Moscow, I-IV 
Table 1. List of new Local Volume dwarf candidates

\begin{tabular}{|c|c|c|c|c|c|c|c|}
\hline \multicolumn{2}{|c|}{ R.A. (1950.0) D. } & $a$ & $b$ & Type & S.B. & Identification & Notes \\
\hline 1 & 2 & 3 & 4 & 5 & 6 & 7 & 8 \\
\hline 001231.6 & -384543 & 0.9 & 0.35 & $\operatorname{Im}$ & $\mathrm{H}$ & AM $0012-384$ & \\
\hline 001253.5 & -214317 & 2.3 & 1.3 & S0 & $\mathrm{H}$ & NGC 59 & $V=382 \mathrm{~km} / \mathrm{s}[1]$ \\
\hline 001300.0 & -322736 & 1.5 & 1.3 & Ir/Sph & $\mathrm{H}$ & FG 11 & granul.,undet.HI [2] \\
\hline 002927.4 & -333230 & 0.9 & 0.8 & $\mathrm{Sph} / \mathrm{Ir}$ & $\mathrm{L}$ & FG 16,AM $0029-333$ & \\
\hline 003252.7 & +361321 & 5: & 3: & Sph & EL & And III & \\
\hline 003443.3 & +475357 & 0.5 & 0.45 & Ir & $\mathrm{L}$ & & distant? \\
\hline 003518.6 & -434646 & 0.6 & 0.4 & $\operatorname{Im}$ & $\mathrm{H}$ & AM $0035-434$ & undet.HI [2] \\
\hline 004256.2 & +374551 & 4: & 3: & Sph & EL & And I & \\
\hline 004651.9 & -182048 & 1.2 & 1.1 & Sph & $\mathrm{L}$ & K 2 & dist.?undet.HI [2] \\
\hline 004756.0 & -201044 & 1.3 & 1.2 & $\mathrm{Sph} / \mathrm{Ir}$ & $\mathrm{L}$ & FG 24 & undet.HI [2] \\
\hline 010603.4 & -382835 & 0.9 & 0.4 & $\operatorname{Im}$ & $\mathrm{H}$ & K 3,AM $0106-382$ & distant?, $V=645[2]$ \\
\hline 011341.9 & +330920 & 4: & $2.5:$ & Sph & EL & And II & \\
\hline 013929.5 & +260657 & 0.7 & 0.4 & Ir & $\mathrm{L}$ & & $V=359[3]$ \\
\hline 014154.0 & +270214 & 1.6 & 0.6 & Ir & $\mathrm{L}$ & & asym., $V=420[3]$ \\
\hline 014353.6 & +263307 & 0.6 & 0.2 & $\mathrm{Ir}$ & VL & & $V=368$ \\
\hline 015230.2 & +274234 & 0.8 & 0.3 & Ir & $\mathrm{L}$ & & comp. N 784 \\
\hline 015718.1 & +283526 & 0.6 & 0.3 & Ir & $\mathrm{L}$ & & comp. N 784 \\
\hline 015722.0 & +673036 & 1.3 & 0.9 & Sph? & EL & & undet.HI [3] \\
\hline $\begin{array}{llll}02 & 02 & 02.4\end{array}$ & +684557 & 2.2 & 1.7 & Ir & $\mathrm{L}$ & Cas 1 & $V=35[4]$ \\
\hline 023139.9 & +222145 & 1.2 & 0.7 & Sph & VL & & \\
\hline 023152.2 & +590942 & 2.4: & 1.0: & Ir & EL & MB 1 & $V=189[5]$ \\
\hline 025154.1 & +583935 & 1.6 & 0.5 & Ir & EL & & \\
\hline 025301.1 & +584237 & 2.0: & $0.3:$ & SB & EL & Cas 2 & $V=112[6]$ \\
\hline 025354.5 & +171525 & 0.6 & 0.4 & $\operatorname{Ir} ?$ & $\mathrm{~L}$ & & distant? \\
\hline 030759.9 & +600928 & 2.8 & 0.8 & Ir? & VL & & refl. neb.? \\
\hline 031853.2 & +623627 & 1.8 & 0.9 & $\mathrm{Ir}$ & $\mathrm{L}$ & & undet.HI [3] \\
\hline 032029.5 & -663004 & 1.2 & 0.4 & Ir & $\mathrm{L}$ & & $\mathrm{EL}$ in $\mathrm{R}$ \\
\hline 032835.2 & +473728 & 1.4 & 0.8 & Ir & $\mathrm{H}$ & UGC 2773 & $V=231[7]$ \\
\hline 033318.9 & -611537 & 1.8 & 0.9 & $\operatorname{Im}$ & EL & FG 82,AM $0333-611$ & resolved \\
\hline 033712.6 & +680250 & 1.0 & 0.5 & Sph? & VL & & \\
\hline 033726.2 & -184942 & 0.7 & 0.6 & $\mathrm{Ir} / \mathrm{Sph}$ & $\mathrm{L}$ & & EL in $R$,dist.? \\
\hline 033726.8 & +193530 & 0.8 & 0.7 & $\mathrm{Ir}$ & $\mathrm{L}$ & & undet.HI [3] \\
\hline 033839.9 & +675257 & $0.4:$ & $0.4:$ & Ir & VL & & \\
\hline 033856.9 & -453052 & 1.5 & 1.5 & $\operatorname{Im}$ & $\mathrm{H}$ & AM $0338-453$ & granulated \\
\hline 034023.7 & +674226 & 2.5 & 1.7 & Ir & VL & & res?,undet.HI [3] \\
\hline 034247.0 & +673057 & 1.0 & 0.6 & $\mathrm{Ir}$ & $\mathrm{L}$ & & distant? \\
\hline 034715.0 & +705634 & 0.9 & 0.4 & $\operatorname{Ir} ?$ & VL & BK 17 & \\
\hline 035847.0 & -623857 & 0.7 & 0.5 & $\operatorname{Im}$ & $\mathrm{H}$ & AM $0358-623$ & \\
\hline 040006.0 & +712000 & 1.1: & 0.9: & $\mathrm{Ir} ?$ & EL & & refl.neb?,br.star \\
\hline 040556.0 & -552721 & 1.6 & 1.0 & $\operatorname{Im}$ & $\mathrm{H}$ & AM $0405-552$ & resolv?,pecul. \\
\hline 041926.7 & +724127 & 3.7 & 2.1 & Sph? & VL & Cam A & comp. N 1560 \\
\hline 043944.4 & +611547 & 0.6 & 0.6 & $\mathrm{Ir} ?$ & VL & & dist?,undet.HI [3] \\
\hline 044511.0 & -360018 & 2.2 & 0.8 & $\mathrm{Im} / \mathrm{Sm}$ & $\mathrm{H}$ & AM $0445-360$ & resolved? \\
\hline 044803.3 & +670102 & 2.2 & 1.1 & $\mathrm{Ir}$ & $\mathrm{L}$ & & $V=75[3]$ \\
\hline 052135.2 & -343713 & 0.55 & 0.4 & $\operatorname{Im}$ & $\mathrm{H}$ & AM $0521-343$ & resolved? \\
\hline 052305.4 & -870514 & 1.4 & 0.7 & $\operatorname{Im}$ & $\mathrm{L}$ & FG154,AM0522 - 870 & resolved? \\
\hline 052749.0 & -873736 & 1.0 & 0.45 & $\mathrm{Im}$ & $\mathrm{L}$ & AM $0528-873$ & \\
\hline 052826.3 & -245444 & 1.7: & $0.3:$ & $\mathrm{Im} / \mathrm{Sm}$ & VL & AM $0528-245$ & \\
\hline 053900.7 & +063928 & 0.7 & 0.5 & $\operatorname{Im} ?$ & $\mathrm{H}$ & & $V=441[8]$ \\
\hline 054725.5 & +025210 & 0.5 & 0.4 & Ir & VL & & shreded \\
\hline 054847.9 & +025348 & 2.1 & 0.5 & Ir & EL & & obscured \\
\hline 060218.4 & -193703 & 1.2 & 0.5 & Ir & $\mathrm{L}$ & & $V=645[1]$ \\
\hline 061251.5 & -513141 & 1.1 & 0.6 & $\operatorname{Im}$ & $\mathrm{L}$ & AM $0612-513$ & resolved? \\
\hline 062416.7 & -261406 & 0.6 & 0.3 & Ir & $\mathrm{H}$ & AM $0624-261$ & $V=495[2]$, res.? \\
\hline
\end{tabular}


Table 1. continued

\begin{tabular}{|c|c|c|c|c|c|c|c|}
\hline 1 & 2 & 3 & 4 & 5 & 6 & 7 & 8 \\
\hline 063755.8 & -404024 & 0.7 & 0.45 & Ir & VL & AM $0637-404$ & $V=272+821[9]$ \\
\hline 063949.0 & +364103 & 1.3 & 0.4 & $\operatorname{Ir} ?$ & $\mathrm{~L}$ & & distant? \\
\hline 070449.9 & -215729 & 1.9 & 1.1 & $\mathrm{Ir}$ & $\mathrm{L}$ & & $V=+737[1]$ \\
\hline 070756.2 & -512308 & 1.4 & 1.1 & Ir & $\mathrm{L}$ & FG 203 & \\
\hline 071741.2 & -571906 & 2.1 & 1.6 & $\mathrm{Im} / \mathrm{Sm}$ & $\mathrm{VL}$ & FG 206,AM $0717-571$ & resolved? \\
\hline 072023.0 & +460610 & 1.1 & 0.4 & Ir & $\mathrm{L}$ & & \\
\hline 072913.1 & +665940 & 3: & $2:$ & Sph & $\mathrm{VL}$ & DDO 44 & undet.HI [10],comp.N 2403 \\
\hline 073150.6 & +421213 & 0.6 & 0.4 & $\mathrm{Ir}$ & $\mathrm{L}$ & & \\
\hline 073720.0 & -691338 & 1.5 & 0.9 & Sph & $\mathrm{VL}$ & FG 219,AM $0737-691$ & \\
\hline 073930.0 & +694109 & 0.6 & 0.2 & $\mathrm{Ir}$ & $\mathrm{L}$ & & distant? \\
\hline 073940.2 & +164047 & 0.9 & 0.5 & $\mathrm{Ir}$ & $\mathrm{H}$ & & comp. U 3974 \\
\hline 074405.4 & +401842 & 0.7 & 0.4 & Ir & $\mathrm{L}$ & & asymmetric \\
\hline 080034.9 & +151703 & 1.0 & 0.5 & Ir & $\mathrm{L}$ & & \\
\hline 082717.0 & -845857 & 1.1 & 1.0 & Ir? & $\mathrm{H}$ & & $V=738[1]$, pec. \\
\hline 084944.1 & +335913 & 2.4: & 1.8: & Sph? & EL & & comp.N 2683 \\
\hline 085216.3 & +334502 & 1.1 & 1.0 & Sph? & EL & & comp.N 2683 \\
\hline 090656.7 & -230951 & 0.45 & 0.35 & $\mathrm{Ir} / \mathrm{Sph}$ & $\mathrm{L}$ & AM $0906-231$ & dist?,undet.HI [9] \\
\hline 090928.5 & -234635 & 0.6 & 0.5 & Sph & $\mathrm{L}$ & & $\mathrm{EL}$ in $\mathrm{R}$ \\
\hline 091015.6 & -240205 & 0.9 & 0.8 & Sph & $\mathrm{L}$ & & EL in $R$,dist? \\
\hline 091218.0 & -232055 & 0.8 & 0.4 & $\operatorname{Im}$ & $\mathrm{L}$ & FG247,AM0912 - 232 & dist?,undet.HI [9] \\
\hline 091248.9 & -254030 & 0.9 & 0.6 & Ir & $\mathrm{L}$ & & $\mathrm{VL}$ in $\mathrm{R}$ \\
\hline 093823.6 & -762141 & 2.1 & 0.8 & Ir & $\mathrm{L}$ & & refl.neb.? \\
\hline 094608.5 & +674425 & 2.4 & 1.8 & Sph & VL & & comp. M 81 \\
\hline 094723.6 & +314126 & 0.5 & 0.3 & $\operatorname{Ir}$ & $\mathrm{H}$ & & UGC 5272B [11] \\
\hline 095003.5 & +293246 & 0.6 & 0.4 & $\mathrm{BCD} ?$ & $\mathrm{H}$ & & \\
\hline 095045.0 & +294057 & 1.1 & 0.6 & Ir & $\mathrm{L}$ & & star projected \\
\hline 095300.8 & +684947 & 2.6 & 2.6 & Sph & $\mathrm{VL}$ & K 61 & comp. M 81 \\
\hline 100025.3 & -055755 & 0.6 & 0.5 & $\mathrm{Ir}$ & $\mathrm{L}$ & & \\
\hline 100118.0 & +664800 & 1.7 & 1.7 & Sph & $\mathrm{VL}$ & DDO 71,K 63 & comp. M 81 \\
\hline 100305.8 & -073020 & 1.9 & 1.3 & Sph & $\mathrm{L}$ & K 65 & comp. N 3115 \\
\hline 100309.0 & +680419 & 2.0 & 1.0 & Sph & $\mathrm{L}$ & K 64,UGC 5442 & comp. M 81 \\
\hline 100522.0 & +304409 & 1.0 & 0.6 & Ir? & $\mathrm{L}$ & MCG $5-24-18$ & v.pecul., dist.? \\
\hline 101237.6 & -443608 & 1.2 & 1.0 & $\mathrm{Sm} ?$ & $\mathrm{H}$ & AM $1012-443$ & \\
\hline 101357.4 & -394423 & 0.9 & 0.5 & Ir & $\mathrm{VL}$ & AM $1013-394$ & $V=263+2982[9]$ \\
\hline 102247.6 & +675432 & 2.0 & 2.0 & Sph & VL & DDO 78 & comp. M 81 \\
\hline 102626.1 & +230157 & 1.0 & 0.15 & $\mathrm{Ir} / \mathrm{S}$ & $\mathrm{VL}$ & & distant? \\
\hline 103100.0 & +661600 & 1.0 & 0.8 & Sph & $\mathrm{VL}$ & $\mathrm{BK} 6 \mathrm{~N}$ & comp. M 81 \\
\hline 103330.1 & +274752 & 0.8 & 0.7 & $\mathrm{Ir}$ & $\mathrm{L}$ & & \\
\hline 104345.8 & +141716 & 1.2 & 1.1 & Ir & $\mathrm{VL}$ & & \\
\hline 104418.1 & +131548 & 1.2 & 0.6 & $\mathrm{Ir}$ & $\mathrm{VL}$ & & near Leo tripl. \\
\hline 104603.6 & +645920 & $2.2:$ & 1.7: & Ir & $\mathrm{VL}$ & UGCA 220 & \\
\hline 104748.8 & +123734 & 1.2: & $0.8:$ & Sph & EL & & near Leo tripl. \\
\hline 105535.3 & +202235 & 0.7 & 0.5 & $\mathrm{Ir}$ & $\mathrm{L}$ & & near edge-on $\mathrm{S}$ \\
\hline 110937.7 & +170132 & 0.9 & 0.4 & $\mathrm{Sph} / \mathrm{Ir}$ & $\mathrm{VL}$ & F 640-3 & undet.HI [12] \\
\hline 111111.2 & -474602 & 0.35 & 0.25 & $\operatorname{Im}$ & $\mathrm{H}$ & AM $1111-474$ & \\
\hline 111122.9 & +113610 & 1.2 & 0.5 & Ir & $\mathrm{VL}$ & & distant ? \\
\hline 111419.1 & -322239 & 0.7 & 0.4 & Sph? & $\mathrm{L}$ & AM $1114-322$ & distant? \\
\hline 112021.2 & +194458 & 0.6 & 0.4 & Ir? & $\mathrm{L}$ & F $570-3$ & undet.HI [12] \\
\hline 112103.8 & +193152 & 0.6 & 0.4 & Sph & $\mathrm{VL}$ & & \\
\hline 112614.5 & +183325 & 1.1 & 0.7 & Ir? & $\mathrm{VL}$ & F $571-10$ & undet.HI [12] \\
\hline 112640.7 & +462323 & 0.8 & 0.5 & $\operatorname{Ir} ?$ & $\mathrm{~L}$ & & \\
\hline 112708.7 & +524054 & 0.8 & 0.7 & Sph? & $\mathrm{VL}$ & K 78 & \\
\hline 113140.1 & +172614 & 0.7 & 0.4 & $\mathrm{Ir}$ & $\mathrm{L}$ & & \\
\hline 113723.0 & +464529 & 0.7 & 0.6 & Sph? & $\mathrm{VL}$ & & \\
\hline
\end{tabular}


Table 1. continued

\begin{tabular}{|c|c|c|c|c|c|c|c|}
\hline 1 & 2 & 3 & 4 & 5 & 6 & 7 & 8 \\
\hline 114433.5 & +435659 & 0.6 & 0.4 & Ir? & $\mathrm{L}$ & & \\
\hline 114601.0 & +561144 & 0.6 & 0.6 & $\operatorname{Ir} ?$ & $\mathrm{~L}$ & & \\
\hline 115127.3 & +165955 & 0.6 & 0.45 & $\operatorname{Ir} ?$ & $\mathrm{~L}$ & & Virgo member? \\
\hline 115210.5 & -331647 & 1.1 & 0.8 & Ir & $\mathrm{L}$ & FG 315,AM $1152-331$ & $V=640[9]$ \\
\hline 115217.9 & +470459 & 0.5 & 0.3 & Ir? & VL & & distant? \\
\hline 115355.1 & -362739 & 0.5 & 0.35 & $\operatorname{Im}$ & $\mathrm{H}$ & & \\
\hline 115537.1 & +490934 & 0.7 & 0.55 & $\mathrm{Im}$ & $\mathrm{H}$ & MCG $8-22-48$ & \\
\hline 115618.5 & +460045 & 1.3 & 0.7 & $\mathrm{Ir}$ & $\mathrm{L}$ & UGCA 259 & $V=1154[10],\{*\}$ \\
\hline 115724.6 & +445950 & 0.6 & 0.6 & Ir? & $\mathrm{L}$ & & \\
\hline 115842.9 & +540301 & 0.6 & 0.15 & Ir & VL & & \\
\hline 115913.5 & +283826 & 0.8 & 0.7 & Ir & $\mathrm{L}$ & & distant? \\
\hline 120056.2 & -251135 & 2.5 & 1.9 & $\mathrm{Im} / \mathrm{Sm}$ & $\mathrm{L}$ & FG 320;AM $1200-251$ & filaments \\
\hline 120252.3 & +435913 & 1.1 & 0.6 & Ir & VL & & \\
\hline 120404.8 & +550238 & 1.0 & 0.25 & Ir & $\mathrm{L}$ & & \\
\hline 120420.5 & +173701 & 0.9 & 0.2 & $\mathrm{Ir}$ & $\mathrm{L}$ & & Virgo member? \\
\hline 120616.1 & +525029 & 0.5 & 0.3 & Ir? & EL & & \\
\hline 121016.9 & +691220 & 0.7 & 0.6 & Sph? & VL & & near br.star \\
\hline 121047.5 & +284144 & 0.6 & 0.3 & Ir & $\mathrm{L}$ & & asymmetric \\
\hline 121051.5 & +301200 & 0.9 & 0.25 & Ir & $\mathrm{H}$ & & $V=152[1],\{*\}$ \\
\hline $1211 \quad 17.1$ & +053754 & 0.6 & 0.4 & $\mathrm{Ir}$ & $\mathrm{L}$ & GR 5 & \\
\hline 121138.9 & +161435 & 1.1 & 1.1 & Sph & VL & K 88,VCC 108 & undet.HI [12] \\
\hline 121517.6 & +284509 & 0.6 & 0.3 & Ir & $\mathrm{L}$ & K 95 & \\
\hline 121558.1 & +285531 & 0.6 & 0.5 & $\mathrm{Sph} / \mathrm{Ir}$ & VL & K 98 & \\
\hline 121638.5 & +480025 & 0.6 & 0.5 & $\mathrm{Sph} / \mathrm{Ir}$ & VL & & \\
\hline 121704.1 & +433950 & 0.4 & 0.3 & Ir & $\mathrm{L}$ & & \\
\hline 121705.3 & +474354 & 0.3 & 0.3 & Sph? & $\mathrm{L}$ & & \\
\hline 121709.5 & +581918 & 0.8 & 0.4 & Ir & VL & & \\
\hline 121813.5 & +471643 & 0.5 & 0.5 & Sph? & $\mathrm{L}$ & & \\
\hline 121913.0 & +381506 & 0.9 & 0.5 & $\mathrm{Ir}$ & VL & K 105 & \\
\hline 121927.0 & +283109 & 0.9 & 0.5 & $\mathrm{Sph} / \mathrm{Ir}$ & VL & & \\
\hline 121937.9 & +400123 & 0.9 & 0.4 & Ir & $\mathrm{L}$ & & asym., dist.? \\
\hline 122015.7 & +081127 & 0.6 & 0.5 & Ir & $\mathrm{L}$ & VCC 584 & \\
\hline 122023.2 & +340623 & 0.4 & 0.3 & $\mathrm{Ir}$ & $\mathrm{L}$ & & \\
\hline 122148.5 & -420057 & 1.0 & 0.5 & $\operatorname{Ir} ?$ & $\mathrm{~L}$ & $\mathrm{AM} 1221-420$ & star w.neb.? \\
\hline 122234.0 & +612020 & 1.0 & 0.7 & $\operatorname{Ir}$ & $\mathrm{L}$ & MCG $10-18-44$ & \\
\hline 122258.6 & +284533 & 1.5 & 0.5 & $\mathrm{Ir}$ & $\mathrm{L}$ & & \\
\hline 122418.6 & +623923 & 1.1 & 0.7 & Ir & $\mathrm{L}$ & UGC 7544 & undet.HI [10] \\
\hline 122419.1 & +132715 & 1.0 & 0.35 & $\mathrm{Sm}$ & $\mathrm{L}$ & DDO 124 & $V=162[10]$ \\
\hline 122520.3 & -370312 & 2.0 & 0.25 & $\mathrm{Im} / \mathrm{Sm} ?$ & $\mathrm{H}$ & AM $1225-370$ & resolved? \\
\hline 122532.4 & +225157 & 0.8 & 0.4 & $\mathrm{Ir}$ & $\mathrm{H}$ & UGC 7584 & \\
\hline 122625.8 & +422720 & 0.8 & 0.45 & $\mathrm{Ir}$ & $\mathrm{L}$ & MCG $7-26-11$ & $V=408[13]$ \\
\hline 122728.2 & +081224 & 1.1 & 0.7 & $\operatorname{Im}$ & $\mathrm{L}$ & UGC 7636 & $V=468[10]$ \\
\hline 122757.5 & +431038 & 1.2 & 0.5 & Ir? & $\mathrm{L}$ & MCG $7-26-12$ & $V=440[13]$ \\
\hline 123058.4 & +333742 & 1.2 & 0.4 & $\mathrm{Ir}$ & $\mathrm{H}$ & MCG $06-28-9$ & granulated \\
\hline 123244.4 & +583945 & 0.5 & 0.3 & $\mathrm{Ir}$ & $\mathrm{L}$ & K 162 & distant? \\
\hline 123456.4 & +390112 & 0.7 & 0.4 & Ir & $\mathrm{H}$ & Arp 211 & \\
\hline 123513.0 & +072242 & 1.2 & 1.0 & $\operatorname{Ir}$ & $\mathrm{L}$ & UGC 7795 & $V=61[10]$ \\
\hline 123809.0 & +473821 & 0.5 & 0.2 & Ir & $\mathrm{L}$ & & \\
\hline 123831.3 & -405303 & 1.7 & 0.4 & $\mathrm{Ir}$ & VL & AM $1238-405$ & \\
\hline 123906.9 & +400513 & 1.1 & 0.7 & Ir & $\mathrm{L}$ & & granulated \\
\hline 124048.0 & +354116 & 0.7 & 0.5 & Ir? & $\mathrm{L}$ & & distant? \\
\hline 124135.8 & +435615 & 0.8 & 0.6 & Ir & $\mathrm{L}$ & & \\
\hline
\end{tabular}


Table 1. continued

\begin{tabular}{|c|c|c|c|c|c|c|c|}
\hline 1 & 2 & 3 & 4 & 5 & 6 & 7 & 8 \\
\hline 124210.0 & +710352 & 1.1 & 0.7 & Ir? & $\mathrm{L}$ & K 195 & \\
\hline 124257.3 & +183425 & 0.9 & 0.7 & $\mathrm{Ir} ?$ & EL & & $\{*\}$ \\
\hline 124337.6 & +621421 & 0.9 & 0.4 & Ir? & VL & & \\
\hline 124526.9 & +044224 & 1.1 & 0.6 & Ir & $\mathrm{H}$ & & distant? \\
\hline 124627.6 & +321433 & 0.7 & 0.7 & $\mathrm{Ir} ?$ & $\mathrm{~L}$ & & \\
\hline 124649.5 & +355305 & 1.7 & 1.0 & Sph & $\mathrm{L}$ & & \\
\hline 124917.8 & +262256 & 0.8 & 0.5 & Ir? & $\mathrm{L}$ & & distant? \\
\hline 125037.8 & +034244 & 0.5 & 0.4 & Ir? & VL & & \\
\hline 125041.3 & +125424 & 0.8 & 0.6 & $\mathrm{Ir}$ & $\mathrm{L}$ & & \\
\hline 125211.5 & -280412 & 0.8 & 0.6 & $\mathrm{Im}$ & $\mathrm{L}$ & AM $1252-280$ & $V=645[14]$ \\
\hline 125303.1 & +331522 & 0.7 & 0.6 & Ir? & $\mathrm{L}$ & & distant? \\
\hline 125414.0 & +121210 & 1.3 & 1.0 & Ir & $\mathrm{L}$ & UGC 8061 & \\
\hline 125607.5 & +180458 & 0.7 & 0.45 & Ir & $\mathrm{L}$ & & Virgo member? \\
\hline 125629.7 & -492108 & 2.2 & 1.6 & $\mathrm{Ir}$ & $\mathrm{L}$ & FG 363 & $V=1895[10],\{*\}$ \\
\hline 125638.7 & +354503 & 0.6 & 0.4 & Ir? & $\mathrm{L}$ & & \\
\hline 125717.1 & -190826 & 1.7 & 0.7 & $\mathrm{Sph} / \mathrm{Ir}$ & VL & & $\mathrm{EL}$ in $\mathrm{R}$ \\
\hline 130015.2 & +221602 & 0.8 & 0.7 & Sph? & VL & F $575-1$ & undet.HI [12] \\
\hline 130032.8 & +262046 & 0.8 & 0.3 & $\mathrm{Ir}$ & $\mathrm{L}$ & F $508-1$ & \\
\hline 130040.5 & -461907 & 1.2: & 0.9: & Sph & $\mathrm{L}$ & FG 367,AM $1300-461$ & comp. N 5128 \\
\hline 130202.1 & +180137 & 1.4 & 0.7 & $\mathrm{Sph} / \mathrm{Ir}$ & VL & F $575-4$ & undet.HI [12] \\
\hline 130209.2 & +270231 & 0.7 & 0.6 & Ir & $\mathrm{L}$ & F $508-\mathrm{v} 1$ & \\
\hline 130212.8 & -394854 & 1.0 & 0.55 & $\mathrm{Ir}$ & $\mathrm{L}$ & & fan-like, $V=619[2]$ \\
\hline 130414.8 & +181608 & 0.6 & 0.45 & Sph? & $\mathrm{L}$ & & \\
\hline 130615.0 & -504446 & 1.9 & 0.4 & Ir & $\mathrm{H}$ & AM $1305-501$ & distant? \\
\hline 130636.8 & +332807 & 0.6 & 0.5 & Ir & $\mathrm{L}$ & & \\
\hline 130703.5 & -231635 & 0.7 & 0.3 & $\mathrm{Im}$ & $\mathrm{H}$ & AM $1307-231$ & \\
\hline 130723.3 & -261943 & 1.0 & 0.8 & $\mathrm{Im}$ & $\mathrm{H}$ & AM $1307-263$ & granulated? \\
\hline 130847.7 & +372639 & 0.9 & 0.6 & $\mathrm{Ir}$ & $\mathrm{L}$ & & \\
\hline 130953.2 & -413401 & 1.0 & 0.6 & Sph? & $\mathrm{VL}$ & & EL in R, comp. N 5128 \\
\hline 131014.6 & -443728 & 2.7 & 1.7 & Sph & $\mathrm{L}$ & FG 373,AM $1310-443$ & comp. N 5128 \\
\hline 131124.8 & +421831 & 0.8 & 0.7 & Sph? & EL & & comp. N 5055 \\
\hline 131202.7 & +365008 & 0.7 & 0.5 & Sph? & $\mathrm{L}$ & & \\
\hline 131316.4 & +414555 & $0.6:$ & $0.6:$ & $\mathrm{Ir} / \mathrm{Sph}$ & EL & & \\
\hline 131507.3 & +443944 & 0.6: & $0.4:$ & Ir? & VL & & \\
\hline 131820.5 & -311605 & 1.3 & 0.6 & $\mathrm{Ir}$ & VL & & $\mathrm{EL}$ in $\mathrm{R}$ \\
\hline 131849.9 & -444805 & 0.6 & 0.4 & Ir? & $\mathrm{L}$ & AM $1318-444$ & \\
\hline 131906.8 & -421620 & 1.5 & 1.1 & Sph & VL & & comp. N 5128 \\
\hline 132007.0 & -331823 & 0.6 & 0.5 & Sph? & $\mathrm{L}$ & CEN 8 & \\
\hline 132031.4 & -285634 & 0.4 & 0.35 & $\mathrm{Ir}$ & EL & & \\
\hline 132148.1 & -304243 & 1.3 & 0.8 & $\operatorname{Im}$ & $\mathrm{H}$ & K 15,AM $1321-304$ & $V=490[14]$ \\
\hline 132220.0 & -372150 & 1.3 & 0.7 & $\mathrm{Ir} / \mathrm{Sph}$ & VL & AM $1321-372$ & near br.star \\
\hline 132237.7 & -290039 & 0.6 & 0.45 & $\mathrm{Ir}$ & $\mathrm{L}$ & & \\
\hline 132429.6 & -450536 & 0.5 & 0.5 & $\mathrm{Ir} / \mathrm{Sph}$ & $\mathrm{L}$ & AM $1324-450$ & \\
\hline 132528.7 & -375437 & 1.6 & 1.1 & Ir? & $\mathrm{L}$ & FG 393,AM $1325-375$ & \\
\hline 132646.8 & +675328 & 1.2 & 0.5 & $\operatorname{Ir}$ & $\mathrm{L}$ & UGC 8509 & undet.HI [10] \\
\hline 133118.6 & +492130 & 1.0 & 0.6 & Ir & $\mathrm{H}$ & MCG $8-25-18$ & \\
\hline 133131.6 & +564526 & 0.6 & 0.4 & Ir? & VL & & \\
\hline 133346.5 & -291900 & $6::$ & 2.5: & Ir & EL & & $\{*\}$ \\
\hline 133552.8 & +492226 & 0.4 & 0.25 & Ir & $\mathrm{L}$ & & \\
\hline 133731.2 & -312647 & 1.4 & 0.6 & $\operatorname{Im} ?$ & $\mathrm{~L}$ & FG 403 & distant? \\
\hline 133903.4 & -445711 & 1.2 & 1.1 & Sph? & $\mathrm{L}$ & AM $1339-445$ & comp. N 5128 \\
\hline 133912.6 & +433231 & 0.8 & 0.6 & Ir? & $\mathrm{L}$ & MCG $07-28-51$ & distant? \\
\hline 134034.6 & -433104 & 0.6 & 0.3 & Sph? & VL & & $\mathrm{EL}$ in $\mathrm{R}$ \\
\hline 134044.0 & -452941 & 0.75 & 0.4 & $\mathrm{Ir}$ & $\mathrm{L}$ & AM $1340-453$ & distant? \\
\hline
\end{tabular}


Table 1. continued

\begin{tabular}{|c|c|c|c|c|c|c|c|}
\hline 1 & 2 & 3 & 4 & 5 & 6 & 7 & 8 \\
\hline 134045.2 & -453922 & 0.5 & 0.5 & $\mathrm{Im}$ & $\mathrm{L}$ & $\mathrm{AM} 1340-453$ & \\
\hline 134124.2 & +434243 & 1.1 & 0.5 & Ir & $\mathrm{H}$ & UGC 8688 & \\
\hline 134313.4 & -452606 & 1.0 & 0.9 & Sph & $\mathrm{L}$ & AM $1343-452$ & comp. N 5128 \\
\hline 134348.7 & -294347 & 1.7 & 0.7 & Sph? & VL & & comp. N 5236 \\
\hline 134521.9 & +393726 & 0.7 & 0.4 & Ir? & $\mathrm{L}$ & & \\
\hline 134522.2 & +332725 & $0.8:$ & $0.7:$ & $\mathrm{Ir}$ & $\mathrm{L}$ & & \\
\hline 134540.5 & -464454 & 1.5: & 1.0: & Sph? & EL & & near br.star \\
\hline 134604.9 & -475721 & 0.5 & 0.4 & $\mathrm{Ir} / \mathrm{Sph}$ & $\mathrm{L}$ & AM $1346-475$ & \\
\hline 134607.8 & +404808 & 0.4 & 0.3 & Ir? & $\mathrm{L}$ & & granulated? \\
\hline 134652.8 & +435054 & 1.0 & 0.4 & $\mathrm{Ir}$ & $\mathrm{L}$ & & distant? \\
\hline 135251.5 & +375542 & 0.45 & 0.3 & $\mathrm{Ir}$ & $\mathrm{L}$ & & \\
\hline 135301.2 & -452447 & 1.1 & 0.7 & Ir & $\mathrm{L}$ & & \\
\hline 135403.7 & +403250 & 0.7 & 0.45 & Ir & $\mathrm{L}$ & & comp. N $5371 ?$ \\
\hline 135721.1 & +523616 & 0.9 & 0.8 & $\mathrm{Ir} ?$ & $\mathrm{~L}$ & UGC 8914 & undet.HI [10], dist? \\
\hline 135954.3 & -465145 & 0.8 & 0.6 & Ir & $\mathrm{L}$ & AM $1359-465$ & \\
\hline 140501.5 & +351809 & 0.6 & 0.5 & $\mathrm{Ir}$ & VL & & \\
\hline 141534.6 & +231821 & 0.8 & 0.3 & Ir & $\mathrm{L}$ & & arched \\
\hline 144048.0 & +500140 & 0.9 & 0.5 & Ir & VL & & patchy \\
\hline 144551.4 & +530231 & 1.1 & 0.7 & $\operatorname{Ir}$ & $\mathrm{L}$ & MCG $9-24-40$ & br.star projected \\
\hline 145713.6 & -513155 & 1.7 & 0.6 & Ir & VL & FG 434 & \\
\hline 150311.2 & -394236 & 0.8 & 0.6 & Ir & $\mathrm{L}$ & FG 438 & distant? \\
\hline 150409.5 & +560324 & 0.9 & 0.7 & Sph? & VL & & \\
\hline 150646.1 & +562703 & 0.7 & 0.5 & Ir? & $\mathrm{L}$ & K 233 & \\
\hline 151159.0 & -225623 & 0.8 & 0.6 & $\operatorname{Im}$ & $\mathrm{L}$ & FG 458,AM $1511-225$ & \\
\hline 152533.2 & -423636 & 1.9 & 0.7 & $\operatorname{Ir}$ & $\mathrm{L}$ & FG 444 & \\
\hline 162223.0 & -595033 & 2.9 & 0.7 & $\mathrm{Sm} ?$ & $\mathrm{~L}$ & ESO $137-$ G27 & \\
\hline 162259.4 & -602053 & 1.6 & 1.0 & $\mathrm{Ir}$ & VL & FG 447 & \\
\hline 175426.1 & +700709 & 0.8 & 0.6 & Sph? & $\mathrm{EL}$ & & comp. N $6503 ?$ \\
\hline 181805.0 & -621744 & 0.9 & 0.6 & Ir & $\mathrm{L}$ & FG 458,AM $1818-622$ & \\
\hline 190920.3 & -615957 & 0.45 & 0.45 & Sph? & $\mathrm{L}$ & AM $1909-615$ & \\
\hline 191617.0 & +635254 & 1.5 & 1.2 & $\mathrm{BCD}$ & $\mathrm{H}$ & NGC 6789 & $V=-157[1],\{*\}$ \\
\hline 200048.0 & -314924 & 1.2: & $0.5:$ & $\mathrm{Ir}$ & VL & FG 492 & \\
\hline 200451.7 & -611230 & 0.9 & 0.6 & $\operatorname{Ir}$ & $\mathrm{L}$ & AM $2004-611$ & \\
\hline 202246.0 & -713423 & 0.8 & 0.4 & $\mathrm{Ir}$ & $\mathrm{L}$ & FG 499 & distant? \\
\hline 202558.8 & -315107 & 0.8 & 0.4 & $\operatorname{Ir}$ & $\mathrm{L}$ & AM $2025-315$ & distant? \\
\hline 202914.4 & +601622 & 1.8 & 0.8 & Ir & VL & UGC 11583 & $V=129[3]$ \\
\hline 202931.9 & +601103 & 1.6 & 0.8 & $\mathrm{Ir} ?$ & VL & & $V=126[3]$ \\
\hline 203033.5 & +603834 & 0.9 & 0.9 & Sph? & VL & & $V=132[3]$ \\
\hline 203330.7 & -692158 & 1.0 & 0.9 & $\mathrm{Ir}$ & $\mathrm{L}$ & AM $2033-692$ & \\
\hline 203346.2 & +605512 & 1.5 & 0.9 & $\operatorname{Ir} ?$ & EL & & undet.HI $[3],\{*\}$ \\
\hline 215421.4 & -603242 & 2.5 & 1.2 & Ir & $\mathrm{L}$ & FG 532,AM $2154-603$ & \\
\hline 220904.6 & -432529 & 0.6 & 0.5 & $\operatorname{Im}$ & $\mathrm{L}$ & AM $2209-432$ & \\
\hline 221925.2 & -483926 & 2.2 & 1.3 & $\operatorname{Ir}$ & $\mathrm{L}$ & FG 545 & \\
\hline 223756.3 & -310340 & 1.6 & 0.8 & Ir? & $\mathrm{L}$ & K $20, \mathrm{FG} 554, \mathrm{AM} 2237-310$ & \\
\hline 230936.4 & -440301 & 4: & $2.2:$ & $\mathrm{Sm} ?$ & $\mathrm{~L}$ & FG 569 & \\
\hline 231146.9 & -435239 & 4.5 & 1.8 & $\mathrm{Ir}$ & $\mathrm{VL}$ & & $\{*\}$ \\
\hline & \multicolumn{6}{|c|}{ [1] Huchra J. (1995) } \\
\hline \multicolumn{8}{|c|}{ [2] Cote S. et al. (1997) } \\
\hline \multicolumn{8}{|c|}{ [3] Huchtmeier W.K. et al. (1997) } \\
\hline \multicolumn{8}{|c|}{ [4] Huchtmeier W.K. et al. (1995) } \\
\hline \multicolumn{8}{|c|}{ [5] Huchtmeier W.K. \& van Driel W. (1996) } \\
\hline
\end{tabular}


Table 1. continued

[6] Kraan-Korteweg R.C. et al. (1994)

[7] Huchra J.P. et al. (1997)

[8] Michel A. \& Huchra J. (1988)

[9] Mattews L.D. et al. (1995)

[10] Huchtmeier W.K. \& Richter O.G. (1989b)

[11] Hopp U.\& Schulte-Ladbeck R.E. (1991)

[12] Schombert J.M. et al. (1992

[13] Garcia A.M. et al. (1994

[14] Mattews L.D. \& Gallagher J.S. (1996)

$\{*\}:$

1156+46. $V=1154 \mathrm{~km} / \mathrm{s}$ and $\mathrm{W}=350 \mathrm{~km} / \mathrm{s}$ contradict to the galaxy morphology.

$1210+30$. In spite of $V=152 \mathrm{~km} / \mathrm{s}$, the galaxy looks like distant.

1242+18. The object looks like an emulsion defect.

1256-49. $\quad V=1895 \mathrm{~km} / \mathrm{s}$ conflicts with the galaxy morphology.

1333-29. Perhaps it is an outer part of $\mathrm{M} 83$.

1916+63. Resolved into stars with the 6-m telescope.

2033+60. May be a reflecting nebula.

2311-43. The system discovered by R.Cannon; near NGC 7531 with $V=1598 \mathrm{~km} / \mathrm{s}$; perhaps a case similar to the LSBD “0110+008” near NGC 428 (Smoker et al. 1996).

Table 2. Other candidates selected in the visual search and rejected after a velocity measurement

\begin{tabular}{|c|c|c|c|c|c|c|c|}
\hline \multicolumn{2}{|c|}{ R.A. (1950.0) D. } & $a$ & $b$ & Type & S.B. & Identification & Notes \\
\hline 1 & 2 & 3 & 4 & 5 & 6 & 7 & 8 \\
\hline 003830.5 & -263228 & 0.6 & 0.3 & Ir & VL & $\mathrm{SC} 27$ & $V=2694[2]$ \\
\hline 014355.7 & +142633 & 1.0 & 0.7 & Ir & $\mathrm{H}$ & UGC 1242 & $V=7389[3]$ \\
\hline 014710.5 & +284003 & 0.7 & 0.5 & Ir & $\mathrm{L}$ & & $V=3841[3]$ \\
\hline 020146.3 & +723023 & 0.8 & 0.8 & Ir & $\mathrm{L}$ & & $V=2918[3]$ \\
\hline 032909.1 & +675636 & 2.0 & 0.8 & Ir & $\mathrm{L}$ & $\mathrm{K} 37=\mathrm{BK} 7$ & $V=1372[3]$ \\
\hline 033344.4 & +672600 & 2.0 & 0.7 & Ir? & $\mathrm{L}$ & BK 8 & $V=1434$ \\
\hline 033825.0 & +680611 & 2.2 & 0.3 & Ir? & $\mathrm{L}$ & BK 12 & $V=1320$ \\
\hline 035322.5 & +690824 & 1.1 & 0.6 & Sph? & $\mathrm{L}$ & BK 19 & $V=1302[3]$ \\
\hline 035934.8 & +712544 & 1.2 & 0.5 & $\mathrm{Ir}$ & VL & BK 21 & $V=1734$ \\
\hline 040644.0 & +703833 & 0.9 & 0.4 & Ir & $\mathrm{L}$ & & $V=1159[3]$ \\
\hline 044640.8 & +670429 & 0.6 & 0.4 & Sph? & $\mathrm{L}$ & & $V=1581[3]$ \\
\hline 130333.1 & -493338 & 1.6 & 0.6 & Ir & $\mathrm{L}$ & & $V=1281$ \\
\hline 134152.9 & -294819 & 0.7 & 0.7 & $\operatorname{Im}$ & $\mathrm{H}$ & FG 405,AM $1341-294$ & $V=4620[2]$ \\
\hline 140637.4 & -300230 & 0.8 & 0.6 & $\operatorname{Im}$ & $\mathrm{H}$ & & $V=2629[2]$ \\
\hline 141600.0 & -450515 & 1.2 & 0.6 & $\operatorname{Im}$ & $\mathrm{H}$ & AM $1415-450$ & $V=1653[2]$ \\
\hline
\end{tabular}

